\title{
RUPTURAS Y CONTINUIDADES EN LOS ROLES Y RELACIONES DE GÉNERO. ESTUDIO SOBRE LAS EXPERIENCIAS Y EXPECTATIVAS FAMILIARES Y LABORALES DE LOS HIJOS E HIJAS DE LA MIGRACIÓN BOLIVIANA EN SALTA (ARGENTINA)
}

\section{BREAKS AND CONTINUITIES IN GENDER ROLES AND RELATIONS. A STUDY OF FAMILY AND LABOR EXPERIENCES AND EXPECTATIONS OF SONS AND DAUGHTERS OF BOLIVIAN MIGRANTS IN SALTA (ARGENTINA)}

Soraya ATAIDE

Resumen: El objetivo de este artículo es analizar las rupturas y continuidades presenten en los roles y relaciones de género de los hijos e hijas de la migración boliviana, particularmente aquella cuyo principal destino es la provincia de Salta. Nos preguntamos sobre el posicionamiento de estos/as jóvenes con respecto a las relaciones conyugales y la maternidad/paternidad, como también aquellas vinculadas a la dimensión laboral. Indagamos en el modo en que se acercan o distancian de las experiencias familiares y laborales de sus padres y madres, quienes migraron desde distintas zonas rurales del departamento de Tarija hacia el noroeste argentino, vinculándose como trabajadores/as de la producción hortícola y que, en su mayoría se encuentran residiendo de forma permanente en la provincia de Salta. A partir de los objetivos planteados, optamos por técnicas cualitativas basadas en entrevistas en profundidad como también observación participante.

* Becaria Posdoctoral del Instituto de Investigaciones en Ciencias Sociales y Humanidades, Consejo Nacional de Investigaciones Científicas y Técnicas. Universidad Nacional de Salta. 
Palabras clave: roles y relaciones de género; hijos e hijas de la migración boliviana; experiencias y expectativas familiares; experiencias y expectativas laborales; mercado de trabajo hortícola.

Abstract: This article is aimed at analyzing the breaks and continuities in gender roles and relations of sons and daughters of Bolivian migrants, particularly those who migrated to Salta province. We were interested in exploring the experiences and expectations young people have with regard to marital life and maternity/paternity, as well as those related to labor. To that end, we studied the way in which these experiences and expectations are close to or far away from the family and labor trajectories of their fathers and mothers. Bolivian peasant men and women that migrated to the northwest of Argentina, became horticultural workers and, for the most part, are permanently living in different horticultural zones of Salta province. According to the stated research objectives, qualitative data collection methods were carried out based on in-depth interviews and participant observation.

Keywords: gender roles and relations; sons and daughters of Bolivian migrants; family experiences and expectations; labor experiences and expectations; horticultural labor market.

\section{INTRODUCCIÓN}

El objetivo de este artículo es analizar las rupturas y continuidades presentes en los roles y relaciones de género de los hijos e hijas de la migración boliviana en la provincia de Salta (noroeste argentino -NOA-). Nos interesó indagar en las experiencias y expectativas que tienen aquellos/as que podemos identificar como nuevas generaciones, de jóvenes, algunos/as nacidos/as en Bolivia y otros en Argentina. Nos preguntamos sobre el posicionamiento de estos/ as jóvenes con respecto a las relaciones conyugales y la maternidad/ paternidad, como también aquellas vinculadas a la dimensión laboral. Indagamos en el modo en que se acercan o distancian de las experiencias familiares y laborales de sus padres y madres, de origen campesino, quienes migraron desde distintas zonas rurales del departamento de Tarija, hacia el NOA. Migrantes que se articularon como trabajadores/as precarios, informales y mal pagos, 
en la actividad agrícola en general y desde las últimas décadas en un mercado de trabajo segmentado por nacionalidad boliviana como es el hortícola, en particular (Ataide, 2017).

Partimos de que la migración constituye uno de los hechos que con mayor fuerza alteran y realinean la vida de las personas (Hondagneu-Sotelo, 2007). La movilidad implica la posibilidad de encontrarse ante destinos donde el sistema de género (De Barbieri, 1992) puede presentar prácticas, símbolos, representaciones, normas $\mathrm{y}$ valores que difieran de aquellos presentes en origen y entonces dar lugar a que los y las migrantes cuestionen aquellas construcciones de género que traen desde sus trayectorias experimentadas previamente en origen. En ciertos casos, el cuestionamiento puede visualizarse en sus hijos e hijas, quienes tienen la posibilidad de revisar críticamente las experiencias familiares y laborales de sus padres y madres (Pedone, 2014).

Justamente, en este estudio reconocemos que los/as jóvenes, muestran en ciertos aspectos de sus experiencias y elecciones, continuidades, pero también rupturas referidas a las expectativas y experiencias de sus padres y madres. Esto puede explicarse por el cambio de contexto espacio temporal de vida, en la reconfiguración de las expectativas que sus padres y madres tienen para con ellos/as, las cuales están presentes en el marco de relaciones intergeneracionales establecidas sobre ciertos roles y relaciones de género hegemónicos en destino.

\section{MARCO TEÓRICO}

La producción científica sobre migraciones ha estado marcada históricamente por su carácter economicista y androcéntrico (Herrera, 2011; Gregorio Gil, 1998). Las investigaciones describían a las mujeres como separadas de la esfera laboral o como si fueran irrelevantes para la misma y por lo tanto, no formaran parte protagónica del hecho migratorio (Ariza, 2007). Los análisis de las migraciones ocultaron a las mujeres migrantes debido a la suposición ampliamente compartida de que ellas (y los niños) migraban para acompañar o para reunirse con los esposos/padres, patriarcas (Mahler y Pessar, 2006, traducción nuestra).

En la década de 1970, investigadoras feministas incorporaron la dimensión de género en los estudios migratorios, advirtiendo que 
existía una desigualdad en las relaciones de poder entre varones y mujeres. En ese marco, se produjo un excesivo foco en las experiencias de las mujeres hasta que, dentro de los estudios postestructuralistas se propuso un abordaje relacional y situacional (Mahler y Pessar, 2006, traducción nuestra). Hacia la década de 1980 se incorporó el enfoque interseccional buscando analizar cómo operan las desigualdades de raza, clase y género. Desde este enfoque las feministas (principalmente aquellas enmarcadas en el feminismo negro) discutieron la categoría de mujer universal reconociendo las múltiples desigualdades que intersectan las experiencias de las mujeres, por ejemplo en contextos migratorios. A su vez, se rechazó la idea de la familia como espacio librado de relaciones de poder y se planteó la existencia del género como estructurador de las relaciones en su interior (Mahler y Pessar, 2006).

Avanzada la década de 1990, ciertas investigaciones visibilizaron la migración de mujeres pioneras, en ciertos contextos geográficos, proceso que sería enmarcado en la llamada feminización cuantitativa y cualitativa de las migraciones. La primera se refería a la creciente presencia de mujeres migrantes en ciertos flujos migratorios, mayormente en sentido Sur-Norte. Mientras que desde la feminización cualitativa se plantearon críticas a la visión androcéntrica del fenómeno migratorio. Lo que se cuestionaba era la representación social afianzada que entendía a los proyectos migratorios internacionales como decisiones y prácticas eminentemente masculinas (Pedone 2008 en Rosas, 2013).

En la investigación que presentamos partimos de que el sistema de género es estructurador de las migraciones (Ariza, 2007) y que la migración es uno de los factores que con mayor fuerza alteran y realinean la vida diaria de las personas que migran (HondagneuSotelo, 2007). A su vez, concebimos al sistema de género como "el conjunto de prácticas, símbolos, representaciones, normas y valores sociales que las sociedades elaboran a partir de la diferencia sexual anátomo-fisiológica" (De Barbieri, 1992 p.114). Dentro de este sistema, la masculinidad y la feminidad son consideradas como las dos diferenciaciones socioculturales primarias de las construcciones de género. Se construyen formas hegemónicas de feminidad y masculinidad y también se definen o moldean las relaciones entre ambos. En ese sentido, el género tiene un carácter relacional dado que no es posible pensar el mundo de las mujeres separado del de los varones, y la mayoría 
de estas relaciones encierran desigualdades en perjuicio de las mujeres (Rosas, 2013).

Dentro de las investigaciones recientes, con enfoque de género en contextos migratorios intra América Latina, se destacan los trabajos que toman como referencia a las migraciones de mujeres pioneras y vinculadas a mercados de trabajo precarios, segmentados por nacionalidad y género como por ejemplo el servicio doméstico remunerado en ciertas metrópolis. Se pueden mencionar los estudios sobre peruanas en Santiago de Chile (Stefoni, 2002), en la ciudad de Córdoba en Argentina (Magliano, 2009) y mujeres peruanas, paraguayas y bolivianas en el AMBA - Área Metropolitana de Buenos Aires - (Courtis y Pacceca, 2010).

Por otra parte, en relación al campo de estudios sobre género y migraciones, Mallimaci Barral (2011 y 2012) advierte que se ha tendido a visibilizar sólo un tipo de proyecto migratorio (el de las mujeres pioneras), en concordancia con las tendencias que el fenómeno migratorio ha tenido en otros contextos geográficos, donde la feminización de las migraciones tanto cuantitativa como cualitativa ha tenido una importante presencia. Asimismo, a partir del análisis del caso de bolivianos/as en Ushuaia, la autora propone superar las categorías binarias utilizadas para explicar las migraciones o bien como laborales o por reunificación familiar. Categorías que se vinculan con la visión tradicional que asocia a los varones con el ámbito público y productivo y las mujeres con el ámbito reproductivo, privado o doméstico (Mallimaci Barral, 2011 y 2012). Desde esta lógica, plantea que los varones aparecen como los actores activos y móviles y por lo tanto los protagonistas de la migración, en cambio las mujeres tendrían el rol de pasivas y en el contexto migratorio, de acompañantes. Siguiendo a la autora, en el caso de las migraciones procedentes de Bolivia, ambas dimensiones, tanto la laboral como la familiar o vincular suelen estar presentes en los varones y las mujeres, aún cuando no aparezcan como la motivación o explicación de la migración (Mallimaci Barral, 2011 y 2012).

Por su parte, Magliano (2013) indagando en clave de género sobre la migración de mujeres bolivianas adultas en la ciudad de Córdoba, identifica los diversos fragmentos que componen sus trayectorias de vida y los roles que asumen en el proyecto migratorio. En los relatos de las/os migrantes reconoce imágenes y representaciones sobre la migrante boliviana, lo cual le permite inferir los sentidos de las 
múltiples presencias en las trayectorias migratorias de las mujeres bolivianas. Justamente, utiliza la noción de "múltiples presencias" para problematizar las categorías binarias que advertían los trabajos antes mencionados en los roles de género para pensar los ámbitos de acción tanto de varones como de mujeres.

Ahora bien, con respecto a las experiencias de hijos e hijas de migrantes existen investigaciones en otros contextos geográficos, particularmente de procesos migratorios con origen en países de América Latina y con destino a países de Europa Occidental (Carrillo, 2005 y Queirolo Palmas, 2005, entre otros). Para nuestro análisis, tomamos el estudio de Pedone (2014) sobre jóvenes reunificados en España donde se analiza las narrativas y prácticas sociales, afectivas y sexuales de chicos y chicas, prestando especial atención a las similitudes y diferencias con respecto a los idearios de sus madres y padres. El trabajo incorpora una dimensión de análisis que resulta interesante para analizar las transformaciones que se producen en las construcciones de género a partir del diálogo intergeneracional. Los/as jóvenes son sujetos con capacidad de transformar las construcciones de sus padres y madres, a partir del cambio de contexto de vida y de la revisión crítica de la experiencia de las generaciones precedentes. Así también, los contextos de destino pueden presentar diferencias sustanciales en relación a los roles y relaciones de género en origen.

Tomando el proceso migratorio de bolivianos/as y paraguayos/as en el AMBA ${ }^{1}$, Gavazzo (2013) investiga las representaciones sociales y sus efectos, tanto sobre las relaciones sociales intergeneracionales como en la autopresentación y las prácticas de los hijos de migrantes bolivianos y paraguayos. Interesa aquí retomar la noción de "generación" que la autora utiliza, en particular en dos de sus sentidos planteados. Uno como una cadena de filiación, genealógica resultando en relaciones de parentesco (abuelo, padre, hijo, nieto), que permite ubicar a quienes estudia como "hijos/as" y un segundo sentido dentro de la estructura etaria general, que la vincula a las distintas etapas del ciclo vital (niñez, adolescencia, juventud y vejez) la cual permite identificar a esos hijos/as además como jóvenes (Gavazzo, 2013).

Asimismo Gavazzo (2013) sostiene que, desde una mirada cientificista se ha tendido a clasificar a la sociedad desde el dato

1 Área Metropolitana de Buenos Aires. 
biológico, es decir, la edad, lo cual condujo a la conceptualización de "la 'juventud' como un período fijo [...] por el que todos vamos pasar, para arribar al mundo adulto (p. 83)" que se percibe como estable, completo e ideal. Inclusive, desde esta mirada de la juventud los adultos se postulan con el poder de controlar y gestionar la vida de esos sujetos. En este sentido, la estructura etaria de una sociedad debe entenderse además como parte de una compleja configuración de las relaciones de poder. En definitiva, considerando estas cuestiones, la autora propone prestar atención a "las prácticas sociales de los jóvenes como sujetos plenos igualmente dotados de capacidad reflexiva y competencia cultural" (Gavazzo, 2013 p. 85).

\section{METODOLOGÍA}

La investigación que aquí presentamos fue realizada con enfoque cualitativo y estrategia etnográfica, utilizando como herramientas de obtención de datos la entrevista en profundidad y la observación participante. El trabajo de investigación fue desarrollado entre 2012 y 2017, dentro de la cual realizamos trabajo de campo tanto en los lugares donde residen las familias (distintos espacios hortícolas de la provincia de Salta, Argentina), como también en los lugares de origen de sus padres y madres (Tolomosa Grande, Iscayachi, San Jacinto, San Andrés, en el departamento de Tarija, Bolivia). Fueron realizadas un total de 47 entrevistas y en su mayoría las personas fueron entrevistadas en más de una ocasión, además de contar con las instancias de observación participante. Esas entrevistas incluyeron a los/ as jóvenes, sus padres y madres (migrantes) y en algunos casos también familiares que permanecen en origen.

A partir del objetivo plantado en la introducción, una primera instancia de la investigación estuvo enfocada en reconstruir las experiencias migratorias y laborales de los padres y madres de los/as jóvenes, quienes migraron desde distintos espacios rurales de Tarija (Bolivia) hacia el NOA, para articularse como trabajadores en la agricultura y en particular en la producción hortícola de la provincia de Salta. En dicha instancia de investigación hemos analizado el caso de 15 varones y 10 mujeres, algunos unidos por vínculos 
conyugales, centrándonos en identificar los principales elementos del sistema de género en origen (identificando los roles y relaciones de género que se presentan como hegemónicos) y cómo operaron en 1) el momento de iniciar la migración y 2) la forma de hacerlo, 3) la edad de formación de la vida conyugal, 3) la edad en la cual se inicia la maternidad/paternidad, como también 4) la inserción laboral en destino (en qué nichos laborales y en qué condiciones). Asimismo, sobre estos/as migrantes nos interesó indagar en las expectativas construidas sobre las experiencias vinculadas a la vida conyugal y laboral de sus hijos e hijas.

En un segundo momento, la investigación focalizó en los/as jóvenes, hijos e hijas de aquellos/as migrantes. Para esto realizamos entrevistas a 15 mujeres y 12 varones, jóvenes de entre diecisiete y veinticinco años cuyas edades se asemejan al momento en el cual sus padres y madres habían iniciado la vida conyugal, como también su experiencia migratoria. Nos interesó indagar en las experiencias y expectativas que tienen los/as jóvenes con respecto al momento de iniciar su vida conyugal y la maternidad/paternidad, como también aquellas vinculadas a la dimensión laboral, observando el modo en que se acercan o distancian de las trayectorias familiares y laborales de sus padres y madres. Para esto también resultó necesario considerar las características del sistema de género de los lugares de destino (en referencia principalmente a cuáles son los nichos laborales en que se ocupan o desean insertarse tanto las mujeres como los varones).

\section{RESULTADOS}

\section{Las trayectorias migratorias familiares y laborales de los/as campesinos/as bolivianos/as en Salta}

La migración de campesinos/as de origen boliviano hacia el NOA puede rastrearse en trabajos previos destacándose las investigaciones de Whiteford (1976), Rutledge (1987), Dandler y Medeiros (1988) entre otros. En aquellos se evidencia la presencia de la migración del campesinado boliviano articulado como mano de obra barata con las principales economías regionales 
del noroeste argentino: la producción de caña de azúcar y tabaco. Hacia la década de 1960, con la crisis en dichas producciones y la incorporación de tecnología ahorradora de mano de obra, la demanda de trabajadores mermó y este flujo migratorio se redireccionó hacia el AMBA y las principales ciudades de la región pampeana, aunque también hacia otros puntos del territorio nacional (Benencia y Karasik, 1995). Sin embargo, muchos continuaron dirigiéndose hacia las provincias del NOA ya sea por cercanía, por la consolidación de redes migratorias, la demanda laboral en ciertos mercados de trabajo, entre otras.

De los casos que hemos analizado, observamos que los proyectos migratorios se construyen en base a un habitus migratorio (Hinojosa Gordonava, 2006). Ciertamente, la migración de campesinos procedentes de Bolivia es reconocida como una estrategia de reproducción social y económica, en este sentido Mallimaci Barral (2012) sostiene que "las migraciones se suponen colaboradoras de la supervivencia familiar, ya sea por las remesas futuras esperadas o por el simple hecho de disminuir la carga familiar" (p. 766).

Asimismo, reconocimos que fueron los varones quienes iniciaron sus trayectorias migratorias previamente a que lo hicieran las mujeres. Realizaron los primeros viajes siendo jóvenes y solteros, a través de redes y cadenas migratorias masculinas que los vincularon con el mercado de trabajo agrícola en el NOA, en actividades caracterizadas por demandar trabajo precario, informal, sacrificado, mal pago y con escasas posibilidades de movilidad ascendente (Ataide, 2017). Migraron junto con hermanos, primos o tíos quienes ya tenían experiencia migratoria y el contacto con algún patrón (dueño de finca), muchas veces oriundos del mismo lugar de origen, a través de los cuales se insertaron como trabajadores. Luego de cierto tiempo de migrar de forma temporaria y circular, algunos de estos varones negociaron la migración de sus parejas mujeres de origen boliviano, para establecerse de forma definitiva en destino. Esas mujeres por su parte debieron negociar su migración en el marco del sistema patriarcal que se produce y reproduce al interior de las familias (Pizarro, 2015). Migrar para ellas estuvo condicionado por la forma legítima de hacerlo para las mujeres en el campesinado tarijeño, es decir se esperaba que lo hicieran junto con un varón, en el marco de una relación conyugal, como ámbito de realización de la maternidad (Ataide, 2019).

Una vez en destino, conjuntamente estas parejas de campesinos 
se emplearon generalmente bajo la figura de la mediería ${ }^{2}$ en distintas fincas hortícolas. Ciertamente, en los lugares de origen de estos migrantes, es decir en el campo tarijeño, tanto varones como mujeres trabajan en la agricultura compartiendo diversas tareas desde muy pequeños, no obstante las tareas domésticas reproductivas están asignadas a las mujeres. Al migrar se observa una reproducción de la división sexual del trabajo en estas parejas de trabajadores migrantes. Inclusive la representación que atribuye a las mujeres bolivianas la condición de "buenas trabajadoras", se desprende de una entrevista realizada a un patrón, dueño de una finca hortícola quien afirmaba sobre una de las trabajadoras bolivianas: "pero si es guapa ella, trabaja más que los varones" (nota del cuaderno de campo, mayo de 2016).

Justamente, la presencia activa de las mujeres bolivianas como trabajadoras desde el primer momento de arribo a destino, permite discutir la idea de que la migración de estas mujeres se explica únicamente en el marco de la reunificación familiar, o simplemente en su rol de acompañantes. En la misma línea en que Mallimaci Barral (2012) cuestiona las categorías binarias para explicar la migración, nuestro caso posibilita comprender la migración de estas mujeres en el marco de relaciones familiares conyugales, pero también donde se espera que ellas trabajen a la par de sus parejas. Por esto, la migración para estas mujeres no puede comprenderse por fuera de la dimensión laboral.

Por su parte, Magliano (2007) plantea que las mujeres bolivianas tienen un papel relevante en el ámbito doméstico y en el productivo, y que son definidas como buenas madres, esposas y también, trabajadoras. Sin embargo, la autora observa que esto no significa la ausencia de relaciones asimétricas de género en ambos ámbitos. En primer lugar existe una subvaloración del trabajo en el hogar, por otro lado las actividades económicas desempeñadas son definidas

2 Refiere a una relación contractual, generalmente de palabra, donde el dueño de la finca provee la tierra, todos los insumos necesarios para el desarrollo del ciclo productivo y toma las decisiones sobre qué, cómo y cuánto se produce; mientras que el mediero o pareja de medieros únicamente su fuerza de trabajo. Este tipo de arreglos pueden concebirse como una relación de dependencia laboral encubierta bajo una supuesta sociedad. En la producción hortícola de la zona estudiada (Gral. Pizarro, Apolinario Saravia y Gral. Güemes) los arreglos suelen pactarse como un porcentaje de la venta de la producción que representa entre un 30 y un 35\% de la misma como ingreso para los medieros. 
como complementarias es decir, son consideradas como una ayuda a la economía del hogar, inclusive por ellas mismas (Magliano, 2007).

En efecto, Magliano (2007) afirma que la familia es el ámbito central de la vida de estas mujeres y que los cambios en las relaciones y roles de género dependen de los contextos pre y pos migratorios y de los modos en que se articulan las identidades de clase, etnia y género (Magliano, 2007). Observa que la gran mayoría de las trabajadoras bolivianas deben afrontar subordinación y exclusión en los contextos familiares y sociales donde persisten desigualdades de género, de clase y de etnia (Magliano, 2007). Asimismo, su inserción laboral en las sociedades de llegada no representa un alivio en sus obligaciones y tareas desempeñadas para muchas de ellas (Magliano, 2007).

Juana, una de las mujeres migrantes entrevistadas, que trabaja junto con su marido en una finca hortícola nos comentaba sobre la organización del trabajo en el cultivo de tomate. Reconocía que ciertas tareas que requieren de mayor esfuerzo físico suelen ser realizadas por los varones, no obstante muchas otras son compartidas:

En el hogar la mujer es la que cocina, lava, todo, a pesar que una va a la quinta, viene uf!, de ese... por ejemplo, en cuestión de cavar, de hacer los hoyos eso hacen ellos, después meter los palos para plantar, en conjunto, igual conjunto hombre y mujer, cuestión de pala eso hacen los varones, para plantar los postes y las varillas, los de la orilla son más gruesos que los de la mitad, eso lo hacen los hombres. Después los alambres, eso lo hacen hombres y mujer. Después la hormoneada ${ }^{3}$, la desbrotada ${ }^{4}$ igual hacemos hombre y mujeres (Entrevista realizada a Juana, en septiembre de 2015, Apolinario Saravia, Salta).

Pero también mencionaba que el trabajo de las mujeres continúa cuando llegan al hogar ya que son las únicas encargadas de las tareas domésticas:

3 Para el cultivo de tomate y para el resto de las producciones realizadas bajo cubierta como el pimiento o la berenjena luego de trasplantar y a medida que la planta crece, se colocan hormonas y se controlan las malezas.

4 Una vez que la planta tiene ya varias hojas, se realiza el desbrote que consiste en recorrerla desde la base, se quitan los brotes axilares, o chupones, que no son hojas. 
Una tiene que volver y seguir haciendo en la casa. ¡Ay! ¡Qué pesado que era antes! ¡Una pesadilla, como para no acordarse! Ahora por ejemplo, tengo mis nenas más grandes, una va a la mañana al colegio y la otra va a la tarde, a la técnica. Ella, la que se queda a la mañana ya cocina, eso es una ayuda muy grande [...] (Entrevista realizada a Juana, Apolinario Saravia, octubre de 2016).

Ese doble trabajo productivo y reproductivo que recuerda Juana ahora se ve aliviado por la presencia de sus hijas mujeres quienes reproducen su rol tradicional femenino. Son ellas las encargadas de cocinar y lavar mientras su madre trabaja junto a su padre. Los hijos varones se encuentran eximidos de las tareas domésticas, aunque en ciertas ocasiones asisten a trabajar a la finca junto con sus padres.

Aquella entrevista con Juana que citamos anteriormente transcurría mientras el marido negociaba con los compradores el precio de los melones producidos por ambos en la finca que arriendan. Sucede que los arreglos comerciales en la actividad son realizados entre varones, en su rol vinculado al espacio público, situación propia de las unidades domésticas que reproducen la filosofía patriarcal tanto en origen como en destino.

Durante décadas estos migrantes residieron en las mismas fincas donde trabajaban, en viviendas precarias, realizadas con maderas, chapas y plásticos, sin luz ni agua potable. En ese marco nacieron los/as hijos/as y también transcurrió la mayor parte de sus vidas. Esos niños/as, actualmente jóvenes, vivieron en el lugar de trabajo de sus padres y madres, trabajadores precarios de un nicho laboral desvalorizado por la población nativa. Esto es, en un contexto de integración subalterna de los padres en lo que concierne a las condiciones materiales de vida.

Actualmente estas familias siguen articuladas con la actividad hortícola, en algunos casos como trabajadoras, bajo la figura de la mediería y en otros como productoras, es decir lograron capitalizarse y poner en producción tierras que adquirieron vía compra o arriendo. Pero más allá de estas diferencias, que refieren a la presencia de movilidad socio económica ascendente, esto no significa una aceptación total por parte de la población nativa, más bien, pueden aparecer como otros subordinados tolerables (Briones, 2008). En definitiva, se tolera su presencia como trabajadores en un nicho laboral desvalorizado como es la horticultura que en distintos puntos del territorio nacional está segmentada por nacionalidad 
boliviana. Y su inserción subordinada suele justificarse a partir de discursos racializantes y etnicizantes, que le asignan la condición de buenos trabajadores, sacrificados y sumisos como si fueran inherentes a su pertenencia nacional (Ataide, 2017).

Los discursos a los que nos referimos se sustentan en la forma en que la migración boliviana en Argentina ha sido configurada como una migración "no deseada" (Domenech, 2011) por no ajustarse con el tipo ideal nacional blanco y europeo (Briones, 2002) desde la visión que contiene la idea de que los argentinos "bajaron de los barcos”. En particular, en Salta, los/as bolivianos/as constituyen los/ as otros/as externos/as y se encuentran ubicados/as en los últimos escalones de la jerarquía social (Ataide, 2019). De acuerdo con Lanusse y Lazzari (2008), el juego de las identidades y diferencias en la provincia de Salta, se despliega entre las categorías de "gaucho", "colla" e "indio". El gaucho como tipo ideal salteño, se constituyó como un sujeto mestizo, más blanco que indio, católico y de herencia hispánica. De esa forma, los grupos de poder local construyeron una autoimagen legitimada en una supuesta condición de superioridad natural (Lanusse y Lazzari, 2008). En este imaginario, los indios del Chaco representan la diferencia interna más irreductible de la salteñidad; el colla, por su parte es asociado a un mestizaje impuro y ligado a las clases bajas, quien es rechazado cuando se "percibe en él marcas de indio; nacional, pero también extranjero (boliviano)" (Lanusse y Lazzari, 2008 p. 207).

Resumidamente, lo anteriormente expuesto refiere a algunos elementos relevantes para comprender el escenario donde transcurrieron las trayectorias de migratorias y laborales de las parejas de migrantes, padres y madres de los/as jóvenes cuyas experiencias abordaremos en el siguiente apartado.

\section{Rupturas y continuidades presentes en las hijas mujeres}

Un primer aspecto que nos plateamos indagar sobre las jóvenes, refiere almomento de iniciarla maternidadyel modo de experimentarla. Reconocemos que son las madres quienes explicitan el deber ser sobre la maternidad de sus hijas. Esto responde al rol tradicional femenino en las mujeres, referido a su función de transmisión de la cultura, además de tener asignadas las tareas reproductoras, de cuidado y por lo tanto, sobre ellas recae el deber ser de madre. 
De todos los casos analizados, sólo dos jóvenes se encontraban en pareja con hijos pequeños. Podemos inferir que existe una tendencia a prolongar los tiempos para iniciar la vida conyugal y también de la maternidad. Esto puede estar asociado a la mayor cantidad de años que presentan en instituciones educativas en Argentina, en relación a sus madres en Bolivia, lo cual también se asocia a la existencia de una situación económica menos apremiante que aquella que vivieron sus madres, pero también a la ausencia de establecimientos educativos en los lugares de origen de las mismas.

Con respecto a las jóvenes madres, al momento de entrevistarlas, ambas estaban dedicadas únicamente al cuidado de sus hijos, mientras los varones reproducían su rol tradicional de proveedores. Esta división sexual del trabajo en la pareja responde a un modelo más cercano al rol que pueden tener las mujeres en destino a diferencia de los lugares de origen, donde es esperable que las mujeres trabajen en el campo, a la par de los varones.

En una ocasión entrevistando en Salta a María Ester (mujer boliviana, de sesenta años) y a su hija Gabriela (de veintidós años) le preguntamos a la joven sobre su vinculación con el trabajo en la producción hortícola a lo cual su madre se apresuró y respondió: “¡No, mimosa salió!” y Gabriela completó: “tengo cinco hermanos varones". Entonces su madre aclaró: "Ella es mamá” (entrevista realizada mayo de 2016, Apolinario Saravia, Salta). Son tres frases cortas que expresan los roles de género esperados tanto para los hijos como para las hijas. Son los varones quienes tienen el mandato de ayudar a su padre en las tareas de la finca mientras que la mujer esta eximida de aquel trabajo pues, por un lado están los hermanos varones para hacerlo y por otro ella "es mamá" y por lo tanto está asignada al cuidado de su bebe. Por otra parte, Gabriela explicita sus expectativas laborales en estrecha correspondencia con los roles de género en destino. Cuando le preguntamos por qué no trabajaba en el campo, como lo había hecho su madre, respondió: "No, nunca fui, si vivía ahí, recorrer pero trabajar, no [...] Yo prefiero trabajar de empleada que ir a la finca. Prefiero trabajar en una tienda, o en mercado, eso sí" (entrevista realizada mayo de 2016, Apolinario Saravia, Salta).

María Ester, madre de Gabriela y otro de sus hijos refuerzan la diferencia entre la expectativa de Gabriela y la representación de las mujeres bolivianas de origen campesino: "Claro [...] más antes, siempre venia gente de Bolivia, mujeres que cosechaban hilvanaban, 
ayudaban al marido". (Entrevista realizada a María Ester, septiembre de 2015, Apolinario Saravia) "Eran parejas que venían, como ellos vinieron (refiriéndose a sus padres) en su juventud, sin hijos, les gustan el trabajo y si vienen, las mujeres trabajan más, se la bancan, hemos visto muchas mujeres así (Entrevista realizada al hermano de Gabriela, Apolinario Saravia, mayo de 2016). Ambos fragmentos reproducen la imagen de la mujer boliviana como buena trabajadora. Por su parte, las experiencias y expectativas de Gabriela se distancian de este ideal y por lo tanto de la trayectoria laboral de su madre y se acercan a los roles asignados para las mujeres en destino y en particular a los nichos laborales feminizados.

Otra joven madre, nos contaba sus expectativas con respecto a su futuro laboral. En su relato caracterizaba la división de tareas con su pareja, el padre de su hija, un joven boliviano a quien conoció en uno de los viajes que realizó con su madre para visitar a la familia en Bolivia. Ella ejerce el rol tradicional de cuidadora mientras su pareja, el de proveedor de los bienes materiales. Esta joven nos expresó su deseo de estudiar y trabajar, pero consideraba únicamente como opciones aquellas que pudiera articular con lo que considera sus obligaciones de madre. Incluso las opciones que planteaba, pueden enmarcarse en trabajos típicamente feminizados: "Y ahora estoy con los chicos, ya cuando me dejen hacer alguito voy a hacer alguito [...] Trabajar y estudiar algunos cursos. Quisiera estudiar maquillaje [...] Quisiera aprender eso y peluquería, me gusta eso. Tres meses será. Así son" (Entrevista realizada a Rosa, marzo de 2016, Apolinario Saravia). La interrupción de las trayectorias educativas en las mujeres jóvenes a partir de la implicancia de asumir responsabilidades de cuidados en la maternidad también aparece en el trabajo de Zenklusen (2018) para el caso los hijos e hijas de migrantes peruanos en la Ciudad de Córdoba (Argentina).

El resto de las hijas mujeres que no se encuentran en pareja residen en el hogar paterno/materno y al momento de nuestra investigación se encontraban terminando la escuela secundaria y proyectando sus estudios o trabajos futuros. Veamos.

Al igual que en los casos anteriores, estas jóvenes tienden a alejarse del trabajo en el campo y por lo tanto de las trayectorias laborales de sus madres. Esto, como mencionamos previamente, se conecta con la mayor cantidad de años que permanecieron en instituciones educativas, en comparación a sus madres, lo cual según lo observado permitió la construcción de proyectos educativos 
a futuro. En cierto modo, esto resulta el camino esperado para las jóvenes en el contexto de destino. ¿Cuáles son esos proyectos?

Hemos observado que las jóvenes optan por estudiar carreras terciarias, por ejemplo profesorados, que se dictan en la ciudad donde viven. Sólo en tres casos se reconoce la decisión de estudiar una carrera universitaria en otra ciudad, correspondiéndose con familias de mayor poder adquisitivo. Inclusive las carreras universitarias seleccionadas se distancian de la actividad agrícola. Consideramos que la elección entre estudiar una carrera de nivel terciario y universitario está vinculada por un lado con las posibilidades materiales de los padres de extender sus obligaciones de manutención económica, que en caso de optar por una carrera universitaria implica a demás, el traslado de sus hijos/as hacia otra ciudad, aumentando los costos en relación a la opción de una carrera terciaria presente en la ciudad donde residen.

Por su parte, las hijas mujeres que no continúan sus trayectorias educativas se articulan con trabajos por fuera de la agricultura, reconociéndose una preferencia por trabajos vinculados al sector de los servicios. Una de estas jóvenes expresaba su percepción negativa en relación al trabajo en el campo: "No me gusta, es muy pesado. No es un trabajo para ir mejor. Las chicas no trabajan en la agricultura. Las que tienen finca si capaz, las que no, no. De vez en cuando ayudan a los padres. No conozco muchas chicas que trabajen". (Entrevista realizada a Rosa, septiembre de 2016, Apolinario Saravia).

De sus palabras podemos inferir que las familias bolivianas (las cuales se dedican especialmente a la producción hortícola), no conciben las tareas que realizan sus hijas en las fincas como trabajo, sino como ayuda, lo cual se distancia del discurso que construye a la mujer boliviana como buena trabajadora.

También Gimena, otra joven nos relataba su preferencia por continuar estudios que la distancien del trabajo de la agricultura, es decir de la trayectoria laboral de su madre.

G: No sé, estoy pensando ¿que será bueno? Es que no sé qué carrera estudiar.

S: Y ¿que hay para estudiar en Saravia?

G: Profesorado no más, pues.

S: ¿De qué?

G: De maestro, de materias así, química, matemática, pero yo para esas materias no soy tan buena. 
La entrevista transcurrió mientras se encontraba trabajando de forma temporal en un comedor. Entonces le consultamos por qué optaba por este trabajo y no la agricultura.

S: ¿Y aprendiste algo acá en la cocina? ¿Te gusta?

G: Digamos a limpiar así. Así no más, digamos a trapear pisos así nomás. Lavar platos, eso nomás. Después cocinar, cocina la señora, ella es la que cocina.

S: ¿Y trabajar en la agricultura? ¿En el maní, no?

G: No $[\ldots]$ Pero yo no voy al maní, no me gusta.

S: ¿Por qué?

G: No me gusta el maní, no me gusta casi el campo.

S: ¿Por qué no te gusta?

G: Y por el sol y otra cosa más, no me gusta casi, no me acostumbro [...] (Entrevista realizada a Gimena, febrero de 2017, San Jacinto, Tarija).

Para las jóvenes entrevistadas sus opciones laborales se asocian con nichos laborales feminizados en destino. Asimismo se distancian de las trayectorias laborales de sus madres, argumentando lo "pesado" del trabajo en la horticultura, y también las escasas posibilidades de movilidad ascendente que lo caracteriza. Inclusive aparece la concepción de que el trabajo en el campo no es un trabajo para las mujeres, expresando que este no sería un nicho laboral femenino. Esto, no solo es manifestado por las jóvenes, sino también por sus madres. La migración, tal como sostiene Zenklusen (2018) puede colaborar en la reconfiguración y redefinición de las expectativas educativas. En nuestro caso a diferencia de la autora citada, no en la propia experiencia de las migrantes sino en las expectativas que tienen para sus hijas.

\section{Rupturas y continuidades de los hijos varones}

En este apartado abordamos las expectativas de padres y madres, con respecto a sus hijos varones y el modo en que se acercan o distancian de sus propias trayectorias familiares y laborales. Asimismo, las analizamos en relación a las expectativas de los mismos jóvenes y la incidencia del nuevo contexto socio económico y del sistema de género en destino.

Con respecto a los proyectos familiares, observamos que en su mayoría estos jóvenes, hijos de bolivianos, en edades similares en 
las cuales sus padres construyeron sus unidades domésticas aún no tienen hijos, ni siquiera se encuentran en pareja, inclusive residen en las mismas viviendas junto con sus padres. En ese sentido observamos cierta ruptura con respecto al proyecto familiar de las generaciones previas. En algunos casos manifestaron su deseo de iniciar una familia propia más adelante, postergando la edad de inicio de la vida conyugal y la paternidad.

En relación a las expectativas laborales que los padres expresan sobre sus hijos reconocemos un posicionamiento crítico sobre el trabajo en la horticultura y la preferencia del alejamiento de los jóvenes en relación a dicho trabajo. María Ester, madre de cinco hijos varones comentaba sobre uno de sus hijos quien había migrado a la Ciudad de Salta para estudiar una carrera terciaria. En el fragmento que citamos podemos inferir su preferencia por el alejamiento de su hijo del trabajo en la producción hortícola cuya explicación radica en las condiciones sacrificadas que lo caracterizan:

Se ha ido a hacer una carrera a Salta (refiriéndose a la Ciudad de Salta), va a trabajar a Salta, ahora estos días esta acá pero ya el domingo se va. Estaba estudiando Seguridad e Higiene, alquila una piecita con otro chango de acá de Saravia. Estaba siguiendo otra carrera $[\ldots]$ ha dejado porque otro de mis changos lo hemos operado y ya no lo pudimos ayudar. Ahora ya ha terminado ya la carrera. Ahora está buscando trabajo, le han quedado en hablar de una empresa. Pero vuelta tiene que ir, tiene pagado un mes más la pieza, no sé qué va hacer, porque el campo es mucho calor, para cosechar debajo de los plásticos, hace un calor que dios mío, una transpiración hasta los ojos puede hacer mal, la vista, jodido, y todos los días, duro, hasta diciembre, cansa [...] (entrevista realizada a María Ester, mayo de 2016, Apolinario Saravia).

Más allá de este caso en particular, la gran mayoría de los jóvenes trabajan en la producción hortícola junto a sus padres, aunque también manifiestan lo sacrificado del trabajo en la horticultura. El resto de los hijos de María Ester residen en el hogar de sus padres, uno se encuentra terminando la escuela secundaria y "ya puso solo, tomate en un invernadero" (entrevista realizada a María Ester, septiembre de 2015, Apolinario Saravia, Salta) en la finca donde trabaja su padre. Otro de los hijos, si bien terminó hace un año la escuela secundaria permanece en Apolinario Saravia trabajando con su padre en la finca. Su padre expresó sus deseos de poder 
ayudarlo para que estudie una carrera en la ciudad de Salta, pero se encuentra analizando sus posibilidades económicas.

En algunos casos, estos jóvenes manifiestan sus deseos de estudiar una carrera universitaria que les permita alejarse del trabajo duro de la horticultura y, en ciertos casos, los jóvenes proyectan permanecer en la producción pero desde un lugar gerencial o administrativo, estudiando ingeniería agronómica. Esto se vincula con las posibilidades económicas de estudiar en la universidad. Pero también significa una continuidad del rol masculino vinculado al ámbito productivo, lo cual no se observa en el caso de las mujeres, como planteamos anteriormente quienes optan por carreras $\mathrm{u}$ oficios en nada vinculados con la producción agrícola.

En un lugar intermedio podemos mencionar el caso de un joven, hijo de un productor de Apolinario Saravia. El padre de este joven nos comentaba sus intenciones de que su hijo pueda estudiar una carrera, en particular Ingeniería Agronómica en la ciudad de Salta, pero por limitaciones económicas no lograron concretar el proyecto. Entonces, el padre y la madre decidieron que estudie otra carrera en Apolinario Saravia. Así fue como el joven comenzó el magisterio, una carrera terciaria que otorga el título de maestro. Lo extraño de este caso radica en el hecho de que el joven se encuentra estudiando una carrera típicamente femenina. Podemos inferir que la elección de estudiar esta carrera resultó de una negociación familiar, lo cual se expresa cuando le consultamos al joven sobre la misma: "en realidad al principio no me gustaba, pero ahora un poco si, mayormente son mujeres las que estudian eso". Lo cierto es que como dijimos, son pocas las carreras que se dictan en los municipios donde residen estos jóvenes y en su mayoría corresponden a profesorados, los cuales también tienen una población mayormente femenina.

Lograr concretar el proyecto de estudiar una carrera universitaria implica la posibilidad económica de la familia de costear el traslado y la vivienda de sus hijos en otra ciudad, lo cual como vimos resulta difícil para estas familias. Pero en este último caso, observamos que es mayor el mandato de darle continuidad a la trayectoria educativa aún cuando no se vincule a la producción agrícola. Ahora bien, el hecho de que este joven se encuentre estudiando no significa que se aleje del trabajo en la finca, ya que continúa colaborando en las tareas de la misma junto a su padre. Este joven también expresó sus expectativas con respecto al trabajo en la horticultura: "en el futuro, 
si Dios quiere, voy a hacer otra cosa [...] No es que no me guste, lo que pasa es que en parte es muy sacrificado y riesgoso".

En definitiva, observamos que, quienes heredan el trabajo de sus padres, ya sea en el rol de trabajadores o bien, en su intento de estudiar una carrera vinculada a la producción agrícola, son los hijos. Lo que está claro es la percepción negativa sobre el trabajo en la producción hortícola, algo que surge del análisis crítico que realizan estos jóvenes sobre las experiencias laborales de sus padres y madres.

Una primera cuestión a resaltar es la mayor cantidad de años de estudio que tienen estos jóvenes en relación a sus padres. También observamos una preferencia por estudiar ingeniería agronómica, una carrera que los mantiene cerca de la producción agrícola pero no del trabajo duro que implica la misma. No obstante, sólo uno de los entrevistados ha culminado sus estudios, otros continúan haciéndolo y varios han desistido. Las limitantes son varias, entre ellas se mencionan la falta de recursos económicos por parte de los padres para mantener a los hijos en los lugares donde estudian, como también las exigencias propias de estudiar una carrera universitaria.

\section{CONCLUSIONES}

En este artículo nos propusimos analizar las rupturas y continuidades presenten en los roles y relaciones de género de los hijos e hijas de la migración boliviana en la provincia de Salta y vinculada a la producción hortícola. Indagamos en las experiencias y expectativas de los/as jóvenes con respecto a la maternidad/ paternidad; la vida conyugal, como también aquellas vinculadas a la dimensión laboral, observando el modo en que se acercan o distancian de las trayectorias familiares y laborales de sus padres y madres. Para el análisis entrevistamos a familias de migrantes que en su mayoría se encuentran viviendo en distintas zonas hortícolas de la provincia.

Lo primero que surge del recorrido del artículo es que, la experiencia migratoria para los varones y mujeres de origen campesino que llegaron a Salta y trabajaron en la horticultura, no ha significado cambios profundos en los roles y relaciones de género para ellos/as mismos, a partir de la migración. Sin embargo, sus 
hijos e hijas muestran rupturas, lo cual se relaciona con el cambio de contexto, pero también con las expectativas que sus padres y madres tienen para sus hijos/as. Entonces existe una reconfiguración y redefinición de las expectativas educativas y laborales de los padres y madres, ya que esperan que sus hijos e hijas transiten por otros mercados laborales diferentes a los que ellos experimentaron.

$\mathrm{Al}$ analizar las experiencias y expectativas familiares de las hijas visualizamos que la mayoría prefiere postergar la edad de la unión conyugal y la maternidad, en comparación con sus madres. En ese sentido encontramos que la mayor cantidad de años en el sistema educativo formal, la posición socio económica menos apremiante que aquella en el cual se encontraban sus padres y madres en Bolivia, la existencia de instituciones educativas en los lugares de residencia, como las características del sistema de género en destino donde es esperable que las jóvenes busquen opciones de continuidad educativa u oficios por fuera del trabajo en el campo, pueden considerarse elementos clave para comprender esta ruptura en relación a las experiencias y expectativas de sus madres. No obstante, reconocimos una continuidad en la división sexual del trabajo que asigna las tareas domésticas y de cuidado a las mujeres.

Asimismo, observamos que estas jóvenes se distancian del trabajo en la agricultura y en particular de la producción hortícola. Ellas se insertan o pretenden hacerlo en otros nichos laborales, algunos también precarios, como el servicio doméstico o la atención al público en comercios, todos mercados de trabajos feminizados en destino. En el caso de las jóvenes que integran familias con mayor poder adquisitivo, son liberadas del trabajo y la preferencia de sus padres y madres es que se dediquen únicamente a culminar sus estudios universitarios. Inclusive las carreras elegidas por ellas, se caracterizan por no tener vinculación directa con la producción agrícola a la cual se dedicaron sus generaciones precedentes durante la mayor parte de sus trayectorias laborales.

Con respecto a los jóvenes, una primera cuestión a resaltar es la mayor cantidad de años de estudio que tienen en relación a sus padres. Al igual que en el caso de las mujeres también se visualiza una postergación en el inicio de la vida conyugal y la paternidad, diferenciándose de la experiencia familiar de sus padres. También creemos que la mayor permanencia en el sistema educativo y la mejor situación socio económica de las familias incide en el modo de proyectar la vida familiar propia. 
Los casos de jóvenes que integran familias con mayor poder adquisitivo se encuentran estudiando la carrera de Ingeniería Agronómica en la Ciudad de Salta o bien, lo han intentado por algunos años. No obstante, sólo uno de los entrevistados ha culminado sus estudios, otros continúan haciéndolo y varios han desistido. Las limitantes son varias, entre ellas podemos mencionar la falta de recursos económicos por parte de sus padres y madres para mantener a los hijos en los lugares donde estudian, como también las exigencias propias de la carrera.

Más allá de estas diversas situaciones, encontramos que, quienes heredan el trabajo en la horticultura son los varones. A pesar de que sus padres y los mismos hijos perciban que el trabajo en dicha actividad es duro y sacrificado, estos jóvenes son quienes colaboran en la finca, en algunos casos suelen permanecer en este mercado de trabajo en forma autónoma y, en caso de tener las posibilidades económicas optan por la carrera (Ingeniería Agronómica) que permita mantenerlos cerca de la actividad aunque desde un lugar gerencial o de toma de decisiones.

\section{REFERENCIAS}

Ariza, M. (2007). Itinerarios de los estudios de género y migración en México. En M. Ariza y A. Portes (Ed.), El país transnacional: migración mexicana y cambio social a través de la frontera. México: UNAM, Instituto de Investigaciones Sociales. Pp.453-515.

Ataide, S. (2017). Inserción desigual de inmigrantes bolivianos en un mercado de trabajo segmentado. Estudio en municipios del este salteño. En Revista Andes $\mathrm{N}^{\circ} 27$. CEPIHA Centro Promocional de Investigaciones en Historia y Antropología. Facultad de Humanidades. Universidad Nacional de Salta.

Ataide, S. (2019). La reproducción de las desigualdades de género en contextos migratorios y laborales. En REMHU, Rev. Interdiscip. Mobil. Hum., Brasília, v. 27, n. 55, abr. p. 181-197

Benencia, R. y Karasik, G. (1995). Inmigración limítrofe: los bolivianos en Buenos Aires. Buenos Aires: Centro Editor de América Latina.

Briones, C. (2002). Mestizaje y blanqueamiento como coordenadas de aboriginalidad y nación en Argentina. En RUNA XXIII. Pp 61-88.

Briones, C. (2008). Formaciones de alteridad: contextos globales, procesos nacionales y provinciales. En Cartografías Argentinas. Políticas indigenistas y formaciones provinciales de alteridad. Buenos Aires: Antropofagia. 
Carrillo, C. (2005). El espejo distante. Construcciones de la migración en los jóvenes hijos e hijas de emigrantes ecuatorianos. En Gioconda Herrera, María Cristina Carrillo y Alicia Torres (editoras) La migración ecuatoriana transnacionalismo, redes e identidades. FLACSO, Sede Ecuador Quito Ecuador. 512p.

Courtis, C. y Pacceca, M. I. (2010). “Género y trayectoria migratoria: mujeres migrantes y trabajo doméstico en el Área Metropolitana de Buenos Aires”. En Papeles de Población, Vol. 16, Núm. 63, enero-marzo. México: Universidad Autónoma del Estado de México.pp. 155-185.

Dandler, J. y Medeiros, C. (1988). “Temporary Migration from Cochabamba, Bolivia to Argentina: Patterns and Impact in SendingAreas”, en P. R. Pessar (Ed.), When Borders don't divide: Labour Migration and Refugee Movements in the Americas. New York: Centre for Migration Studies, pp. 8-41.

De Barbieri, T. (1992). Sobre la categoría de género: una introducción teórico-metodológica. En Fin de Siglo, Género y cambio civilizatorio. Chile: ISIS Internacional, Ediciones de las Mujeres $\mathrm{N}^{\circ} 17$.

Domenech, E. (2011). Crónica de una 'amenaza' anunciada. Inmigración e 'ilegalidad': visiones de Estado en la Argentina contem-poránea. En La construcción social del sujeto migrante en América Latina: prácticas, representaciones y categorías / coordinado por Bela Feldman-Bianco. [et al]. - Quito: FLACSO, Sede Ecuador: Consejo Latinoamericano de Ciencias Sociales, CLACSO: Universidad Alberto Hurtado. 366 p.

Gavazzo, N. (2013). No soy de aquí, ni soy de allá. Alterización y categorías de identificación en la generación de los hijos de inmigrantes bolivianos y paraguayos. En Claroscuro. Revista del Centro de Estudios de Diversidad Cultural 12. Pp 73-95.

Gregorio Gil, C. (1998). Migración Femenina. Su impacto en las relaciones de género. Madrid: Ediciones Narcea. 288p.

Herrera, G. (2011). Género y migración internacional en la experiencia latinoamericana. De la visibilización del campo a una presencia selectiva. En Política y Sociedad, 2012, Vol. 49 Núm. 1: 35-4.

Hinojosa Gordonava, A. (2006). La transnacionalización de los procesos migratorios en Bolivia. En Laruta Bustillos, Carlos et. al., Las Migraciones bolivianas. Opiniones y análisis 83. Fundemos Ediciones. La Paz. Bolivia, pp 137-178.

Hondagneu-Sotelo, P. (2007). La incorporación del género a la migración: "no solo para feministas - ni solo para la familia". En M. Ariza y A. Portes (Ed.), El país transnacional: migración mexicana y cambio social a través de la frontera. México: UNAM, Instituto de Investigaciones Sociales, Pp 423-453.

Lanusse, P. y Lazzari, A. (2008). Salteñidad y pueblos indígenas: continuidad y cambio en identidades y moralidades., En Briones, Claudia (comp.) Cartografías Argentinas. Políticas indigenistas y formaciones provinciales 
de alteridad, Editorial Antropofagia, Buenos Aires.

Magliano, M. (2007). Mujeres, migración y roles de género. El caso de la migración boliviana hacia Argentina. En XI Jornadas Interescuelas Departamentos de Historia. Departamento de Historia Facultad de Filosofía y Letras. San Miguel de Tucumán:Universidad de Tucumán.

Magliano, M. (2009). Migración, género y desigualdad social. La migración de mujeres bolivianas hacia Argentina. En Estudios feministas, Florianópolis 17(2) 344 mayo-agosto. pp 349-367.

Magliano, M. (2013). Los significados de vivir múltiples presencias: Mujeres bolivianas en Argentina. En Revista Migraciones Internacionales. Vol. 7, $\mathrm{n}^{\circ}$ 1, Enero-junio. pp 165-195.

Mallimaci Barral, M. (2011). Migraciones y géneros. Formas de narrar los movimientos por parte de migrantes bolivianos/as en Argentina. En Estudos Feministas, Florianópolis, 19 (3): 392, setembro-dezembro.

Mallimaci Barral, M. (2012). Dossier. Revisitando la relación entre géneros y migraciones. Resultados de una investigación en Argentina. En Revista Mora. pp 161-176.

Mahler, S. y Pessar, P. R. (2006). Gender Matters: Ethnographers Bring Gender from the Periphery toward the Core of Migration Studies. En IMR Volume 40 Number 1 Spring: Pp.28-63.

Pedone, C. (2011). Familias en movimiento. El abordaje teórico-metodológico del transnacionalismo familiar latinoamericano en el debate académico español. En Revista latinoamericana de estudios de la familia. Vol. 3, enero-diciembre. pp. 223-244.

Pedone, C. (2014). Rupturas y continuidades de los roles de género en contextos migratorios transnacionales. Relatos sobre sexualidad y salud reproductiva de los hijos e hijas de la inmigración ecuatoriana en Cataluña. En Papeles del CEIC, vol. 2014/2, n ${ }^{\circ} 111$, CEIC (Centro de Estudios sobre la Identidad Colectiva). Universidad del País Vasco, Recuperado de: http:// dx.doi.org/10.1387/pceic. 12968

Pizarro, C. (2015). Intersection of Inequalities: Migratory Trajectories, Labor Experiences, and Family Life of Bolivian Women on the Outskirts of Buenos Aires and Córdoba. En C. Pizarro (Ed.), Bolivian Labor Immigrants Experiences in Argentina.New York City:Lexington Books. Pp. 51-66.

Queirolo Palmas, L. (2005). Entre ciudadanía, discriminación e integración subalterna. Jóvenes latinos en Génova. En G. Herrera, M. C. Carrillo y A. Torres (Ed.), La migración ecuatoriana transnacionalismo, redes e identidades pp.397-432.

Rosas, C. (2013). Las participaciones migratorias de los varones y las concesiones del género. Veracruzanos (Mexicanos) en Chicago y peruanos en Buenos Aires. En Revista Científica. Vol.XVII N¹, Otoño. Sección Artículos: UCES p 111-131.

Rutledge, I. (1987). Cambio agrario e integración. El desarrollo del capitalismo 
en Jujuy 1550-1960. Buenos Aires: Centro de Investigaciones en Ciencias Sociales.

Stefoni, C. (2002). Mujeres inmigrantes peruanas en Chile. En Papeles de Población 8 (33). Toluca: Universidad Autónoma del Estado de México. Whiteford, S. (1976). Migration in Context: A Systematic Historical Approach to the Study of Breakdown Before Urbanization. Vol. 62. $\mathrm{N}^{\circ}$. 3, pp. 147-162.

Zenklusen, D. (2018). Trayectorias situadas. Una aproximación a la experiencia de jóvenes peruanos/as que residen en Córdoba, Argentina, desde una perspectiva de género. En Magliano, M.J (comp). Entre márgenes, intersticios e intersecciones: desafíos pendientes entre género y migraciones. Buenos Aires: Teseopress, pp.25-54. 